\title{
Technical public representation for health risk assessments in a highly urbanized region
}

\author{
F. Falcone ${ }^{1}$, M. Dolen ${ }^{2}$ \\ ${ }^{1}$ Institute for Environmental Engineering Research, \\ Villanova University; USA \\ ${ }^{2}$ College of Nursing, Villanova University; USA
}

\section{Abstract}

A successful Health Risk Assessment (HRA) associated with substantial subsurface hydrocarbon contamination in a densely populated $\&$ industrialized region of a major city requires focused and continuous interaction with numerous segments of the affected population. Without such interaction, vocal established public organizations can stall progress and impede new and/or emerging development because of a lack of clear understanding. To provide such understanding and to help insure project success as measured through continual progress, a Technical Advisory Group (TAG) must be established to represent the public in technical aspects of the HRA process. It must also be responsible for providing tailored educational services to a wide range of culturally diverse population sectors in the community to insure the HRA results are clearly understood and accepted. The TAG MUST BE viewed as unbiased, technically competent, widely experienced and capable of discussing complex technical issues at levels of education commensurate with the affected population. This paper presents such a specific project located in Philadelphia, Pennsylvania; USA, which began in January, 1998 and is still on-going.

\section{Introduction}

The definition, evaluation and eventual remediation of complex environmental problems require a wide range of interaction among many diverse and generally exclusive professional disciplines. Such is the case for the environmental problem discussed in this paper. Political, cultural, social, legal, scientific and technical aspects must all be addressed in a "holistic" approach rather than in a linear approach. This requires not only functional interaction but mutual trust and respect. Here in lies the deepest challenge in defining and achieving success. 
The specific environmental problem currently under evaluation is very "real" and very "close" to human health issues because it deals with the confirmed existence of substantial hydrocarbon contamination ( 2 million gallons) floating freely on groundwater under a very highly urbanized section of Philadelphia, Pennsylvania, USA. The problem is very "real" and very "close" because there is a suspected link between this type of contamination and human and ecological health issues in that region.

Discussed in its broadest terms, the PLUME is defined as the existence of this hydrocarbon contamination below ground level and floating on groundwater. It has resulted in a surface area of approximately 115 acres which emits volatile organic compounds (VOCs) on a continual basis. Depending on the permeability and porosity of the soil above the plume, a certain portion of these VOCs can escape directly through the soil up into the atmosphere. Also, these VOCs can enter residential basements, commercial \& industrial structures, open pit construction sites, schools \& institutional facilities, storm drains, sewer lines and other utility pathways. A map of Philadelphia and the affected region is provided as chart \#1.

\section{Subsurface contamination}

The nature of the hydrocarbon contamination is such that it contains, among other compounds, the following four VOCs: Benzene, Toluene, Ethyl Benzene and Xylene. These are commonly found in gasoline and referred to as BTEX. Of these BTEX chemicals, Benzene is a known carcinogen. The others may cause different types of health problems in humans and ecological systems. The demographic region above and near the PLUME is characterized by densely populated neighborhoods with families commonly dwelling in row homes, both heavy and light industry, vibrant commercial centers, highly active transportation networks and a very active and ethnically diverse population and social structure. Given the extent of the contamination, the possibility of its expansion, the known dangers of Benzene, the presence of other VOCs and the highly urbanized and complex nature of the political region affected, it was decided by the U.S. Federal Government to conduct a detailed Health Risk Assessment (HRA). The basic purpose of this HRA is to answer the questions, "What are the potential health risks to the population living and working above and around the plume?" And, "Where are zones of highest contamination concentration which might affect the population?" Through detailed mathematical modeling, HRAs of this type are designed to quantify the health risks. If the results of the HRA show that at least one more cancer case will develop in the region per 1 million residents, then there is a defined health risk which must be investigated further. If the results show that at least one more cancer case will develop in the region per 10,000 residents, then a defined health risk is confirmed and immediate action is required. Under this latter circumstance, numerous actions can occur including relocation of all residents from a defined area to a different area, removal and remediation of soil, the establishment of zoning restrictions for further use of the contaminated area, etc. 


\section{Establishment of a Technical Advisory Group (TAG)}

Although these remedial steps seem appropriate, straight forward and in the public interest, implementation can be extremely difficult given the urbanized nature of the affected region. Recognizing the complex nature of the issue and in order to insure that public concerns are fully addressed, the U.S. Federal Government decided to select a "Technical Advisory Group (TAG)" to represent the public throughout the HRA process. Basically, the TAG is responsible to insure that all public concerns are fully addressed in the HRA, that data used to draw conclusions adequately represents the existing subsurface situation and that the final HRA results show clearly and accurately the health risk associated with the contamination under investigation. In addition, the TAG is responsible for providing public outreach and educational services to the community so that residents clearly understand what an HRA is and what it isn't. And, so that the public can develop a level of acceptance of the HRA results when they become available. It is most interesting to note that, in selecting a TAG, the U.S. Federal Government searched the ACADEMIC community in the greater Philadelphia region. They excluded the private technical consulting community and other federal, state or local agencies because, it was thought that the public would view these other types of organizations as "biased" in a particular direction. Whereas, the academic community is viewed as unbiased, technically sound and capable of explaining complex environmental issues and problems to a public with a very wide range of levels of education. Through discussions with representatives from the U.S. Government involved with this project, it was learned that this selected $T A G$ is very unique in its makeup and totally different from other TAGs throughout the USA addressing other similar problems. In other similar situations, usually only one individual with specific technical expertise serves as the TAG. Here, in the Philadelphia project, 4 individuals with various technical expertise make up the TAG. This approach seems to have been highly successful. And, therefore, can serve as a model for other similar problems in highly urbanized regions.

\subsection{Major Stakeholders}

Regarding the origin of the contamination under consideration, the U.S. Federal Government, through its Defense Supply Center in Philadelphia (DSCP), and the Sun Oil Company (SUN) were named as "major stakeholders".

In January 1998, these major stakeholders (DSCP \& SUN) were named in a "Consent Order" and ordered to clean up the subsurface hydrocarbon plume. Funds were provided by the U.S. Federal Government for selection of the TAG. But, the community was tasked with providing a structure through which the TAG would be selected and would operate. The community responded by organizing a community group to act in this regard. This community group was formed from the Restoration Advisory Board (RAB) of DCSP, which already existed and from the Citizens Action Community Advisory Panel (CAP) of SUN which also already existed, (see chart \#1). These two groups, served to form the public group through which the TAG would provide its services. Consequently, 
the Citizens Action Working Group (CAWG) was formed to interact with the TAG. Although the establishment of these various organizations may seem cumbersome and bureaucratic, this level of complexity in organizational structure reflects the complex nature of the region and the environmental problems under consideration. To date, since January 1998, this organizational structure has proven to be effective in achieving the stated goals.

\section{Chart \#1 - TAG Selection and Organizational Structure}

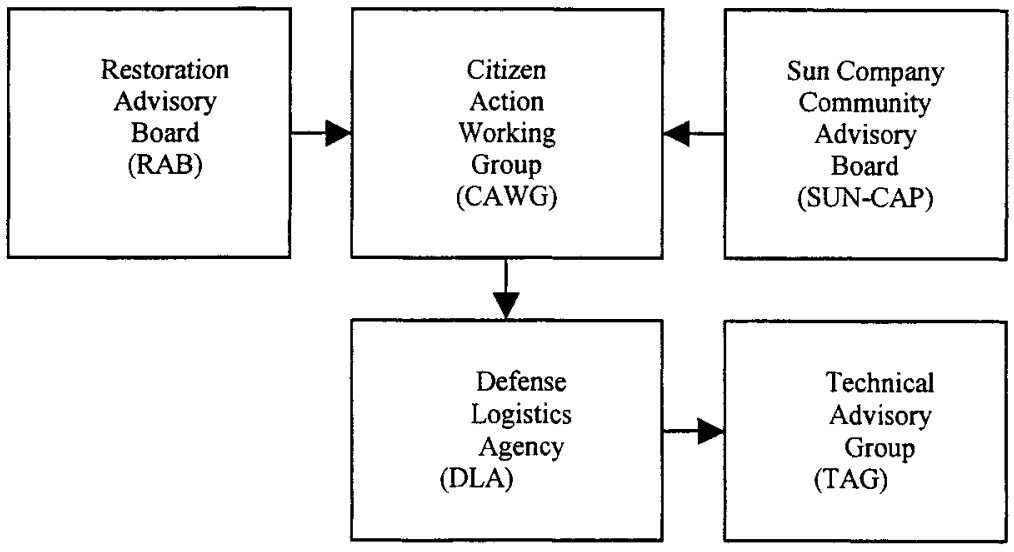

\subsection{Make up of the TAG}

Through the organized selection process, the Villanova University Institute of Environmental Engineering Research (IEER) along with a well-known and wellrespected toxicologist from the region was selected to form the TAG. Because of the range of technical expertise available through TAG members, it was deemed that this group would fully and accurately represent the concerns of the public throughout the process and would provide the educational services to the community as necessary. Technically, the TAG includes a Civil Engineer with deep experience in environmental issues and specific expertise in hydraulics and hydrology, a Chemical Engineer with a full understanding of chemical contaminants and their interaction among themselves and with surrounding materials, a Ph.D.Registered Nurse with specific experience in public health issues and a toxicologist with a long list of detailed and specific experience in Risk Assessment projects and experience with the BTEX VOCs issues discussed above. It is perceived by the CAWG as an unbiased, third party, technical opinion group.

\subsection{Responsibilities of the TAG}

In its specific interaction with the public, the TAG and the CAWG worked together to define exactly how these technical and public outreach services 
would be provided. In this context, the specific responsibilities of the TAG are to:

1.) Work with the CAWG in facilitating the public's understanding of the process and outcomes of the HRA and to learn from the public, through the CAWG, their concerns and questions about the plume.

2.) Review, evaluate and make recommendations to the CAWG, on a routine basis regarding the data gathered and assumptions made by the authors of the HRA.

3.) Represent the CAWG at all stakeholder meetings. It should be noted that due to the extreme complexity of this problem and its associated potential health risks, it has become a very visible and highly publicized issue in Philadelphia. Because of this, these stakeholder meetings now also include representatives from the Philadelphia Health Department, the Philadelphia Housing Authority, the Pennsylvania Department of Environmental Protection and the U.S. Environmental Protection Agency.

4.) Provide ongoing education through community outreach programs that have been developed in full conjunction with the CAWG such that this education is targeted directly to the educational levels of the public.

\subsection{Interaction with the TAG}

Although the TAG is viewed as the "teacher" and "educator", true interaction developed as a result of both groups, CAWG and TAG, learning from each other. At its heart, success in this project is based on the fact that the TAG views itself as a group of technical teachers, advisors AND LEARNERS!! Education, throughout this project, has been a reciprocal effort by the CAWG and the TAG to raise each other's awareness of the problems as seen by each group and by the public in general. The CAWG educated the TAG regarding previous efforts by local and outside groups to address various health issues. Only through a full and clear understanding of the cultural and social issues affecting the residents and workers in the region, could the TAG understand the technical impact of the results of the HRA.

The public's previous experience with highly technical information presented as entire reports resulted in their experiencing "information overload" and often becoming confused with the technical terminology not properly and adequately explained. The first few CAWG / TAG meetings were devoted to the process and components of a health risk assessment (HRA). The methods and routes of exposure, fate and transport of chemicals, toxicity data and technical principles were discussed in such a way as to foster questions and interaction. The goal at these initial meetings was to share key information such that all participants understood clearly the issues under consideration. Each subsequent meeting was structured to give updated information, solicit questions and comments and finally to provide recommendations and advice. Since the members of the CAWG had been working with other stakeholders for longer than they had been working with the TAG, the CAWG members' insight into the relationships among the stakeholders was useful. 


\section{Technical focus of the TAG}

The major and most focused work of the TAG was reviewing and evaluating the technical reports of the HRA as well as the numerous previous reports including all technical background data. This was done from a civil, environmental and chemical engineering, toxicological and health perspective and was enhanced by the varied educational and practical backgrounds and experiences of the TAG. This activity was guided by the need to assure the CAWG that the final HRA would be technically sound, representative of good science, compliant with federal, state and local regulatory guidelines and was complete and correct. The review of the first draft revealed that more work needed to be done in the area of exposure assessment. The various exposure pathway scenarios proposed did not represent all the possibilities for residents and workers who might come in contact with the plume, either directly (for example, workers excavating over the plume) or indirectly (for example, children playing in potentially contaminated soil in nearby residential areas). Since the health and safety of these individuals is of paramount importance and is at the heart of the HRA, these "exposure scenarios" required further definition to encompass all possible exposure pathways. In addition, the extent of the plume was also not adequately delineated in the first draft of the HRA. Therefore, further detailed work was required in this area. Specifically, more subsurface data needed to be gathered to more adequately define the extent of the plume. This detailed and thorough review of the first draft of the HRA focused on exposure scenarios, the nature and extent of contamination, procedures and computations. And, it resulted in a TAG recommendation to revise the project WORK PLAN and redefine the project with a new WORK PLAN that totally reflected the well-founded concerns of the public. The requirement for this new WORK PLAN was accepted by the CAWG, the U.S. Federal Government and all other stakeholders. With the revised and expanded work plan in progress, the TAG visited the actual sites where soil gas data was being collected. The extent of soil gas contamination is used to determine the extent of the subsurface oil contamination and, therefore, the extent of the actual plume. Photographs from these visits were shared with the CAWG and used in subsequent public meetings. The TAG reviewed the findings of every phase of the revised draft HRA, made further comments and recommendations. At the request of the Pennsylvania Department of Environmental Protection (PADEP), the TAG also reviewed the Indoor Air Sampling Work Plan as well as the methodologies for using geotechnical data in vapor intrusion modeling. Although this information seems peripheral to the overall project, it serves as justification of the TAG's approach to technical representation. Once again, without the TAG's full understanding of all related issues, PADEP may not have decided to request this ancillary information from the TAG.The TAG attended all meetings of the stakeholders. Given the nature of the technical issues present for discussion, the TAG, at times, called such meetings. On a somewhat routine basis, present at these meetings were DSCP, SUN, RAB, the City of Philadelphia Health Department, the City of Philadelphia Housing Authority, several attorneys representing interested individuals, other invited environmental engineering firms, the Pennsylvania Department of 
Environmental Protection, the U.S. Army Corps of Engineers, the U.S. Environmental Protection Agency and the engineering firms producing the HRA. Although numerous entities are represented at these meetings which might lead to the conclusion that "nothing would be accomplished", this is usually not the situation. Due to well thought out organization and control, these meetings are productive and beneficial. They serve the public well in that when the final HRA is presented, it is anticipated that the public will accept it, in part, because they've been fully represented throughout the entire process. This method and approach proved to be extremely useful in moving the process forward. The purpose of the TAG at these meetings is to represent the community and its interests as envisioned by the CAWG.

\subsection{Peripheral TAG responsibilities}

In addition to these stakeholder meetings, the TAG has also attended Restoration Advisory Board (RAB) meetings as both observers and presenters. The RAB requested that the TAG attend and provide an educational overview to the public "at large" regarding the nature of HRAs. It became evident through participation in these RAB meetings that the public "at large" had no understanding of the HRA process or its projected goals. It was widely held among numerous sectors of the public that the HRA would definitively pinpoint sources of health concerns such that any individual living and/or working in the community exposed to contamination from the plume, would clearly be in a position to link his or her personal and specific medical problems directly to contaminants emitting from the plume. This is, of course, neither the goal nor the intent of the HRA. Through these meetings, the TAG educated participants as to the real value of the HRA; namely, that if cancer exposure is increased as discussed above, action would be taken by federal or state agencies that would benefit the public "in general". But, it was clearly pointed out to the public that the HRA is not a personal document that can be used as a source of diagnosis for a specific individual's illnesses. Without the TAG's acceptance by the public as a credible and unbiased third party technical representative, this education would not have been effective as it has been thus far in the process.

\subsection{Communication issues}

The process thus far, 1998 - present, has been a learning effort for all involved. But, through the TAG's continual and unwavering focus on interaction, it is felt that progress has been steady and that the community has been well represented. Most certainly, the forthcoming next draft of the HRA will represent the public's concerns to a much greater level of detail than did the first draft. Regarding problems during the process, they are "communications" based. Not unlike other major efforts requiring input from numerous different organizations with different viewpoints, communication techniques are usually at the center of problems that can stall progress. This project has been no exception to that unstated but well-known rule. 


\section{Public outreach services}

As identified above, providing community outreach education is another responsibility of the TAG. This role began with educating the CAWG and has become more important as the HRA process proceeded. Due to the need for a new revised WORK PLAN, the date of project completion was extended and the local communities became disenchanted with the process, worried and angry. In order to address this problem, decisions were made with the CAWG regarding the mechanics, target audiences and processes for informing the community of the activities to date. The SUN communications department, having done prior community outreach on this subject in the region, was most helpful to the TAG in identifying channels, formats, materials and methods for strategies.

The two primary communities in the City which were most affected are ethnically, socially, racially, culturally, educationally and economically extremely different. Therefore, education approaches to each must be substantially different. It should be noted that substantial differences were needed in these two communities BUT NOT TOTALLY DIFFERENT approaches. These two communities immediately affected by the plume, are very different in all aspects. For purposes of this paper, we can refer to them as Packer Park Homes (PPH), an economically "middle class" series of interlocking neighborhoods on the edges of the plume. This community is represented by 2 members on the CAWG. These 2 representatives, through their own local community organizations, have been very responsible and outstanding in keeping citizens in their communities apprised of the progress of the HRA. The second community will be referred to as Passyunk Homes (PH), a federally funded residential low-income housing community located directly over the plume. Most of the PH residents have lived in that community for over 40 years and had their own residents' council. PH also had 2 representatives on the CAWG. However, it was very interesting to note that their participation at meetings was, at best, sporadic. As a result of this sporadic participation at CAWG / TAG meetings, PH residents were not aware of the HRA process nor of the progress being achieved. Clearly, providing educational services to each of these groups would require different approaches and different communication strategies.

\subsection{Passyunk Homes (PH)}

Since virtually all of the $\mathrm{PH}$ residents are African-American, an AfricanAmerican community organizing firm, hired by SUN, was used to identify, recruit and mobilize opinion leaders in $\mathrm{PH}$. These leaders were willing to work not only with the TAG but also with other smaller groups in PH. In preparation for their work in the community, educational sessions were offered by the TAG. The basic strategy was to educate a few individuals designated as "opinion leaders" with fairly formal classroom type training focused on the HRA process. Then, they, in turn, would pass this education on to others within the $\mathrm{PH}$ community. These initial education sessions were based on the "Train the Trainer" model and utilized principles of adult learning. Identifying and focusing on what PH residents were most concerned about, the TAG elicited information 
regarding the residents' concerns about the plume. As expected, these concerns were primarily health related. Through these discussions, the TAG could visualize the depth of health concerns and the extent of incorrect information that was widely held to be true by PH residents. This "information sharing" allowed the TAG to devise the second educational strategy for PH residents, an in-depth explanation of what a risk assessment is, the exposure pathways, fate and transport of chemicals, and the physical extent of the plume. These explanations were augmented by twenty 24"X36" drawings of the assessment process along with maps of the plume, handouts and physical teaching aids such as jars of water and oil to simulate the plume as it floats on groundwater. These sessions set the tone for the opinion leaders' understanding and stimulated many additional questions. Evaluation of this activity, obtained through completed survey forms, reflected total acceptance by PH residents in that their level of understanding was very substantially increased and outdated ideas regarding the nature of the plume and the goals of the HRA were effectively dispelled.

The final strategy for $\mathrm{PH}$ residents was the materials and media methods for reaching the audience. This was a four-fold brochure written with input from the opinion leaders. This brochure covered the basic questions about the plume. Among others, these questions included the following: "Where is the plume? What is in it? Can the chemicals in the plume cause me harm? Where can I get more information? The opinion leaders were given this brochure to hand out to other PH residents. A Power Point presentation about the plume based on the brochure was added to computers in the Residents' Learning Center.

\subsection{Packer Park Homes (PPH)}

For Packer Park Homes (PPH), the two members of the CAWG representing this area have been very effective in keeping PPH citizens apprised of the HRA process and progress through their civic association. Therefore, it was decided "Train the Trainer" sessions, similar to those provided for PH residents, was neither warranted nor desired AT THAT TIME. The brochure, developed and based on the educational program for $\mathrm{PH}$ residents was rewritten with input from the CAWG given to the PPH representatives and distributed through them, to residents in that community.

\subsection{Community wide strategy}

The next strategy for community outreach for all of the affected and potentially affected communities was to offer two "Plume Fairs". Similar to a health fair, this activity had been recommended and developed by SUN and had been used as an effective "outreach tool" prior to the TAG's working with the CAWG. The TAG participated in two plume fairs, for the two communities (PH \& PPH) that were held in separate locations on the same day. Having the fair at different times and in different locations allowed for greater access by various interested groups. Neither Plume Fair was restrictive in nature. Instead, they were both fully open events designed to encourage information sharing and interaction. For each Plume Fair, stakeholders set up booths in which they provided written 
literature and computer generated visual materials about their organizations and their connection to the environmental problem under consideration. Each booth had stakeholder representatives who responded to the many questions of the attendees. Organizations represented were SUN, DSCP, the City of Philadelphia Water Department, the City of Philadelphia Health Department, the TAG, PADEP, as well as the various environmental firms carrying out the health risk assessment. And, the interactive nature of these events which strived to foster "cross communication" among all interested parties proved to be extremely successful in bringing all residents to a point of clear understanding and acceptance regarding the plume and current and planned activities designed to reduce potential health risks.

\section{Conclusions and what's different about this project}

The establishment of this type of TAG for community representation for this type of environmental problem by the U.S. Federal Government has proven to be singularly unique when compared to other TAGs established throughout the USA. The success, thus far, in technical public representation for this Philadelphia project can be attributed, in large part, to partnership and interaction fostered by the TAG. Listed below are the issues:

\section{Extensive complex hydrocarbon contamination}

Base HRA on actual, real data gathered at points of primary concern and not Solely on easily available data.

Ethnically, culturally, socially, racially, educationally and economically diverse communities exposed to the same contamination

Provide education to residents tailored to their specific needs and levels of education.

Working with various diverse established community groups and government agencies

Provide input through one group, the CAWG, which, in turn provides representation throughout various communities.

Evaluation of a wide variety of technical issues involving many technical disciplines

Build a TAG Team that includes professional expertise in all technical areas without exceeding a manageable and affordable number of professionals.

Provide all necessary technical representation services and public outreach services within established budgets.

Refrain from holding regularly scheduled meetings and sessions. But, only hold such meetings when necessary. Progress, under such a scenario, is measurable and visible. 